\title{
Identification and characterization of a barley gene controlling cuticle wax formation
}

\author{
Gerasimova S. ${ }^{1,2 *}$, Kolosovskaya E. ${ }^{1,2}$, Hertig C. ${ }^{3}$, Hiekel S. ${ }^{3}$, Korotkova A. ${ }^{1}$, \\ Doroshkov A. ${ }^{1,2}$, Kukoeva T. ${ }^{1}$, Domrachev D. ${ }^{4}$, Kochetov A. ${ }^{1,2}$, Kumlehn J. ${ }^{3}$, \\ Khlestkina E. ${ }^{1,2,5}$ \\ ${ }^{1}$ Institute of Cytology and Genetics, SB RAS, Novosibirsk, Russia \\ ${ }^{2}$ Novosibirsk State University, Novosibirsk, Russia \\ ${ }^{3}$ Leibniz Institute of Plant Genetics and Crop Plant Research (IPK), Gatersleben, Germany \\ ${ }^{4}$ Novosibirsk Institute of Organic Chemistry, SB RAS, Novosibirsk, Russia \\ ${ }^{5}$ N.I. Vavilov All-Russian Institute of Plant Genetic Resources (VIR), St. Petersburg, Russia \\ *e-mail:gerson@bionet.nsc.ru
}

The deposition of lipid-based polymers at the surface of plant epidermis affects a large set of diverse traits in crops. The group of WAX INDUCER1/SHINE1 (WIN1/SHN1)like transcription factor genes belonging to the APETALA2/ETHYLENE RESPONSIVE ELEMENT-BINDING PROTEIN (AP2/EREBP) gene family has been shown to be involved in the regulation of lipid biosynthesis and cuticle formation in plants. Taking a reverse genetic approach, the present study aims to reveal functions of few WIN1/ SHN1-like genes in barley. The site-directed knockout of four WIN1/SHN1-like genes in spring barley (cv. Golden Promise) was performed using Cas 9 endonuclease targeted to a region conserved across all four related genes. An analysis of T0 and T1 mutants revealed different combinations of mutated target genes in individual plants. Various mutant phenotypes were identified in T1 families, which included a deficiency in cuticle wax deposition at the stem and leaf surface. Co-segregation analysis revealed this wax-deficient phenotype to be associated with mutations in one of target genes, namely HvWin 1. The cuticle wax measurement and scanning electron microscopy showed that independent mutations in the Winl gene invariably lead to a significant reduction in total wax deposition at the leave blades, while stem and leaf sheaths of such mutants were almost entirely free of wax. These results suggest an organ-specific transcriptional regulation of cuticle wax accumulation and Winl-mediated activation of cuticle wax component biosynthesis or transport in barley. Cuticle wax-deficient barley mutants constitute a useful experimental model for further studies on the transcriptional regulation of cuticle organization and the role of cuticle composition in the context of biotic and abiotic stress resiliance.

Acknowledgements: The study is supported by the RSF (16-14-00086). 